\title{
Origin of compartmentalization in food webs
}

\author{
R. Guimerà, ${ }^{1,2,3,4,11}$ D. B. Stouffer, ${ }^{5}$ M. Sales-Pardo, ${ }^{1,2,4,6}$ E. A. Leicht, ${ }^{7}$ M. E. J. Newman, ${ }^{8,9}$ \\ AND L. A. N. Amaral ${ }^{1,2,10}$ \\ ${ }^{1}$ Department of Chemical and Biological Engineering, Northwestern University, Evanston, Illinois 60208 USA \\ ${ }^{2}$ Northwestern Institute on Complex Systems (NICO), Northwestern University, Evanston, Illinois 60208 USA \\ ${ }^{3}$ Institució Catalana de Recerca i Estudis Avançats (ICREA), Universitat Rovira i Virgili, Tarragona, 43007 Tarragona, \\ Catalonia, Spain \\ ${ }^{4}$ Department of Chemical Engineering, Universitat Rovira i Virgili, Tarragona, 43007 Tarragona, Catalonia, Spain \\ ${ }^{5}$ Integrative Ecology Group, Estación Biológica de Doñana, CSIC, 41092 Sevilla, Spain \\ ${ }^{6}$ Northwestern University Clinical and Translational Science Institute (NUCATS), Northwestern University, \\ Chicago, Illinois 60611 USA \\ ${ }^{7}$ Department of Mechanical and Aeronautical Engineering University of California, Davis, California 95616 USA \\ ${ }^{8}$ Department of Physics, University of Michigan, Ann Arbor, Michigan 48109 USA \\ ${ }^{9}$ Center for the Study of Complex Systems, University of Michigan, Ann Arbor, Michigan 48109 USA \\ ${ }^{10}$ Howard Hughes Medical Institute (HHMI), Northwestern University, Evanston, Illinois 60208 USA
}

\begin{abstract}
The response of an ecosystem to perturbations is mediated by both antagonistic and facilitative interactions between species. It is thought that a community's resilience depends crucially on the food web - the network of trophic interactions - and on the food web's degree of compartmentalization. Despite its ecological importance, compartmentalization and the mechanisms that give rise to it remain poorly understood. Here we investigate several definitions of compartments, propose ways to understand the ecological meaning of these definitions, and quantify the degree of compartmentalization of empirical food webs. We find that the compartmentalization observed in empirical food webs can be accounted for solely by the niche organization of species and their diets. By uncovering connections between compartmentalization and species' diet contiguity, our findings help us understand which perturbations can result in fragmentation of the food web and which can lead to catastrophic effects. Additionally, we show that the composition of compartments can be used to address the long-standing question of what determines the ecological niche of a species.
\end{abstract}

Key words: compartmentalization; compartments; ecological networks; food web patterns; food web structure; food webs; niche; modularity.

\section{INTRODUCTION}

Ecosystems face threats arising from extinctions, invasive species, and the accumulation of persistent contaminants (Srinivasan et al. 2007, Ng et al. 2008, Stouffer et al. 2008). The response of an ecosystem to these threats is mediated by both antagonistic and facilitative interactions between species. One important set of interactions is referred to as the food web (Cohen et al. 1990, Pimm 2002, Pascual and Dunne 2006), that is, the network of trophic interactions between species. Despite the existence of other types of interactions, such as mutualisms (Bascompte and Jordano 2007), the topological properties of food webs, including the distribution of number of prey and predators per species (Camacho et al. 2002, Dunne et al. 2002, Stouffer et al. 2005), structural motifs (Bascompte and Melián 2005, Camacho et al. 2007, Stouffer et al. 2007, Kondoh 2008), the contiguity of species diets or intervality (Cohen 1977, Sugihara 1984, Williams and Martinez

Manuscript received 30 June 2009; revised 3 December 2009; accepted 1 February 2010. Corresponding Editor: D. A. Spiller.

${ }^{11}$ E-mail: roger.guimera@urv.cat
2000, 2008, Cattin et al. 2004, Stouffer et al. 2006, Allesina et al. 2008), and compartmentalization (May 1972, Pimm 1979, Pimm and Lawton 1980, Yodzis 1982, Girvan and Newman 2002, Krause et al. 2003, Allesina and Pascual 2009, Rezende et al. 2009), are thought to play a central role in the propagation of ecological perturbations (Pimm 1979).

Among these properties, compartmentalization is believed to be particularly important. Compartmentalization refers to the existence of groups of species that have a higher probability of interacting with one another than with other species in the food web (May 1972, Girvan and Newman 2002, Krause et al. 2003). In the propagation of ecological perturbations (Teng and McCann 2004), as in other dynamical processes taking place on complex networks (Arenas et al. 2006), the existence of compartments results in a separation of time scales: if food webs are truly compartmentalized, it is thought that perturbations will propagate far faster within compartments than between compartments (May 1972, Melián and Bascompte 2002).

An idea that has been less frequently considered is that compartmentalization could potentially determine the effect of perturbations that alter the structure of the 
food web itself. On one hand, perturbations that reduce food web compartmentalization may make ecosystems more prone to system-wide catastrophic events by blurring the boundaries between compartments. On the other hand, perturbations that increase compartmentalization may lead to de facto fragmentation of the ecosystem. The potential effects of excessive compartmentalization are significant enough that Pimm (1979) concluded that "species interactions should not be arranged in tight compartments."

It has recently been established that empirical food webs are more compartmentalized than the random null hypothesis in which each species preys with equal probability on every other species (Krause et al. 2003, Rezende et al. 2009). Allesina and Pascual have also shown that group-based models provide a very good description of the observed trophic interactions in real food webs (Allesina and Pascual 2009). In this context, two important questions remain to be definitively answered: (1) What truly constitutes a compartment within a food web (and what is the relationship between traditional compartments and the groups defined by Allesina and Pascual)? And (2) which factors explain the observed compartmentalization? Here we address both of these questions and then discuss the type of scenarios that are likely to change a food web's compartmentalization and thus its resilience to perturbation.

To tackle these questions, we make use of the most accurate tools in the literature for the identification and analysis of compartments within complex networks (Newman and Girvan 2004, Danon et al. 2005, Arenas et al. 2007, Guimerà et al. 2007, Leicht and Newman 2008). We systematically apply these tools for the first time to quantify the compartmentalized structure of food webs and understand the mechanistic origin of such compartmentalization. We consider three complementary definitions of compartment, encompassing the classic concept of compartment (Krause et al. 2003, Rezende et al. 2009) and the more recent definition of "group" by Allesina and Pascual (2009). Furthermore, we analyze the mechanisms behind observed compartmentalization in ten food webs from a variety of environments. This analysis enables us to draw general conclusions regarding the origin of compartmentalization in food webs.

We find that the compartmentalization observed in empirical food webs can be accounted for solely by the niche organization of species and their diets. In addition, we show that one can use the composition of real food webs' compartments to quantitatively address the longstanding question of what properties of a species determine its ecological niche.

\section{Methods}

\section{Compartment identification}

The search for compartments in complex networks is also often referred to as analysis of "community structure" (Newman and Girvan 2004) owing to origins in social science literature. Similarly, compartments are also referred to as "topological modules" in the general study of complex networks (Danon et al. 2005). A widely used approach to the identification of compartments in complex networks is to define a quality function, the modularity, that is high for "meaningful" partitions of a network into compartments and zero for typical (random) partitions (Newman and Girvan 2004). Despite some limitations (Fortunato and Barthélemy 2007, Sales-Pardo et al. 2007), modularity maximization is the most accurate current method for identifying compartments. Here, we describe in detail the modularity functions we use for the identification of compartments in food webs.

The definition of a modularity function depends on how the network is represented. Food webs are directed networks in that trophic interactions occur between predators and prey that play distinct roles in the interaction. In a directed representation, the links can be thought of as arrows going from prey to predator in the direction of mass flow. As a simplification, however, one can disregard the direction of the interactions and create an alternate representation which is referred to as an undirected network. In a food web, the predators and prey are still connected but the links are no longer arrows; given the undirected representation of a food web it is not possible to determine which species are predators and which prey.

The modularity $M_{\mathrm{U}}(P)$ of a partition $P$ of an undirected network is defined as the fraction of links within compartments minus the expected fraction of such links (Newman and Girvan 2004). The expected fraction of links within compartments is evaluated assuming that the probability that nodes $i$ and $j$ are connected is $k_{i} k_{j} / 2 L$, where $k_{i}$ is the number of links of node $i$ and $L$ is the total number of links in the network. Therefore, the modularity is as follows (Newman and Girvan 2004):

$$
M_{\mathrm{U}}(P)=\frac{1}{2 L} \sum_{i j}\left[A_{i j}-\frac{k_{i} k_{j}}{2 L}\right] \delta_{m_{i}, m_{j}}
$$

where $A$ is the adjacency matrix of the network (that is, $A_{i j}=1$ if there is a link between $i$ and $j$ and $A_{i j}=0$ otherwise), $m_{i}$ is the compartment of node $i$, and $\delta$ is Kronecker's delta $\left(\delta_{a, b}=1\right.$ if $a=b$ and $\delta_{a, b}=0$ otherwise). This modularity function has been recently used to identify compartments in food webs by Rezende et al. (2009).

The simplest generalization of Eq. 1 to directed networks is the following (Arenas et al. 2007, Leicht and Newman 2008):

$$
M_{\mathrm{D}}(P)=\frac{1}{L} \sum_{i j}\left[A_{i j}-\frac{k_{i}^{\text {in }} k_{j}^{\text {out }}}{L}\right] \delta_{m_{i} m_{j}} .
$$

Now, $k_{i}^{\text {in }}$ and $k_{j}^{\text {out }}$ are, respectively, the number of incoming and outgoing links of node $i$. Following the 
convention mentioned above, the outgoing links of a node indicate the predators of the species, the incoming links its prey, and $A_{i j}=1$ if species $i$ preys on species $j$.

An alternative approach for the directed case is to consider the outgoing (incoming) links of each node, and then build compartments comprising nodes with similar outgoing (incoming) links. The modularity $M_{\mathrm{O}}(P)$ of a partition $P$ of a directed network, according to the outgoing links, is as follows (Guimerà et al. 2007):

$$
M_{\mathrm{O}}(P)=\sum_{i j}\left[\frac{c_{i j}^{\text {out }}}{\sum_{l} k_{l}^{\text {in }}\left(k_{l}^{\text {in }}-1\right)}-\frac{k_{i}^{\text {out }} k_{j}^{\text {out }}}{\left(\sum_{l} k_{l}^{\text {in }}\right)^{2}}\right] \delta_{m_{i} m_{j}}
$$

where $c_{i j}^{\text {out }}$ is the number of outgoing links that $i$ and $j$ have in common. As before, the second term inside the brackets comes from the random expectation for $c_{i j}^{\text {out }}$.

The goal of a module identification algorithm is to find the partition $P^{*}$ that yields the largest modularity (note that the number $N_{\mathrm{C}}$ of compartments is only constrained to be $N_{\mathrm{C}} \leq S$, but is otherwise selected by the optimization algorithm). Due to the large number of possible partitions of a network and to the rugged structure of the modularity landscape, it is necessary to use heuristic algorithms to identify maximum-modularity partitions. We use a spectral algorithm to maximize modularity Eq. 2 (Leicht and Newman 2008) and simulated annealing to maximize the modularity Eq. 3 (Guimerà and Amaral 2005, Guimera et al. 2007).

\section{Compartment homogeneity}

We quantify the homogeneity of compartments in three ways which we refer to as trophic-level, sink, and niche homogeneity. Trophic-level homogeneity measures the extent to which compartments comprise species with contiguous trophic levels. To calculate trophic-level homogeneity within compartments, we start by sorting species according to their trophic level $l_{i}$, which we calculate as follows (Levine 1980):

$$
l_{i}=1+\frac{\sum_{j} A_{i j} l_{j}}{\sum_{j} A_{i j}} .
$$

We then count the number $B^{*}$ of boundaries, in that ordering, between the observed compartments (see Appendix A: Fig. A1). For example, if we have two compartments, one compartment comprising the species with lowest trophic levels and another comprising the species with the highest trophic levels, then $B^{*}=1$. In general, if compartments are perfectly homogeneous, the number of boundaries is $B^{*}=N_{\mathrm{C}}-1$ (where $N_{\mathrm{C}}$ is, as before, the number of compartments). If the compartments are less homogeneous in terms of the trophic levels of the species they comprise, the number of boundaries will be larger.
We define trophic-level homogeneity $H_{\mathrm{t}}$ as

$$
H_{\mathrm{t}}=\frac{\langle B\rangle-B^{*}}{\sigma_{B}}
$$

where $\langle B\rangle$ is the average number of boundaries between compartments for the ensemble of all possible partitions of the species (with the same number of compartments and the same compartment sizes as the observed partitions), and $\sigma_{B}$ is the standard deviation of the same quantity. Both of these quantities can be calculated analytically (see Appendix A for the full derivation).

Niche homogeneity measures to what extent compartments correspond to contiguous regions of the niche space. We define niche homogeneity $H_{\mathrm{n}}$ in the same way as trophic-level homogeneity, but counting the boundaries in the niche space, rather than the trophic-level space. (In principle, this can only be done with model networks, for which the niche value of each species is known; we discuss the issue of empirical proxies for niche value later.)

Sink homogeneity measures to what extent compartments correspond to sink food webs within the food web. For each species $i$, we first find the set $T_{i}$ of species that belong to $i$ 's sink food web (which is made up of $i$ 's prey, the prey of $i$ 's prey, and so on until no new species are encountered). We then count the number $o_{i}^{*}$ of species that belong to both $T_{i}$ and species $i$ 's compartment $C_{i}$. We define sink homogeneity $H_{\mathrm{s}}$ as

$$
H_{\mathrm{s}}=\sum_{i=1}^{S} \frac{o_{i}^{*}-\left\langle o_{i}\right\rangle}{\sigma_{o_{i}}}
$$

where $\left\langle o_{i}\right\rangle$ is the average number of species that belong to both $T_{i}$ and $C_{i}$ for the ensemble of all partitions of the species (with the same number of compartments and the same compartment sizes as the observed partitions), and $\sigma_{o_{i}}$ is the standard deviation of the same quantity. (As before, these quantities can be calculated exactly; see Appendix A for the full derivation.)

\section{Food-web models}

We consider two models to generate food webs: the niche model (Williams and Martinez 2000) and the generalization of the niche model by Stouffer et al. (2006). To generate a food web using the niche model (Williams and Martinez 2000), one considers $S$ species and assigns, to each of them, a niche value $n_{i}$ drawn from a uniform distribution in the interval $[0,1]$. Each predator $j$ then preys on the species in a range $r_{j}=n_{j} x$ of the niche axis, where $x$ is drawn from a beta-distribution $p(x)=\beta(1-x)^{(\beta-1)}$ and $\beta=\left(S^{2} / 2 L\right)-1$, where $L$ is the total number of trophic interactions in the network. The center of the range $r_{j}$ is selected uniformly at random in the interval $\left[r_{j} / 2, n_{j}\right]$. All species $i$ whose niche values $n_{i}$ fall within this range are considered prey of species $j$. This implies that, in the niche model, all predators' diets are contiguous within the dimension provided by the niche axis. 
TABLE 1. Food webs and their properties from the literature.

\begin{tabular}{lllrr}
\hline \hline \multicolumn{1}{c}{ Food web } & \multicolumn{1}{c}{ Reference } & $S$ & $z$ & \multicolumn{1}{c}{$c$} \\
\hline Benguela & Yodzis (1998) & 29 & 7.0 & 0.95 \\
Bridge Brook Lake & Havens (1992) & 25 & 4.3 & 0.96 \\
Caribbean Reef & Opitz (1996) & 50 & 11.1 & 0.75 \\
Chesapeake & Baird and Ulanowicz (1989) & 31 & 2.2 & 0.96 \\
Coachella & Polis (1991) & 29 & 8.8 & 0.88 \\
Little Rock & Martinez (1991) & 92 & 79 & 0.92 \\
Northeast U.S. Shelf & Link (2002) & 25 & 7.7 & 0.78 \\
Skipwith & Warren (1989) & 48 & 4.9 & 0.52 \\
St. Marks & Christian and Luczkovich (1999) & 0.82 \\
St. Martin & Goldwasser and Roughgarden (1993) & 42 & 4.9 & 0.87 \\
\hline
\end{tabular}

Notes: Properties are number of species $S$, linkage density $z$, and diet contiguity $c$. Diet contiguity $c$ is estimated as detailed in the Methods.

To generate a food web using the generalized niche model (see Appendix C: Fig. C1; Stouffer et al. 2006), one also assigns to each species a niche value drawn from a uniform distribution in the interval $[0,1]$, as in the niche model. The generalized niche model, however, allows for tunable prey contiguity. First, the reduced range $r_{j}^{\prime}$ for predator $j$ is set to $r_{j}^{\prime}=c \times r_{j}=c \times n_{j} x$, where $c$ is a fixed parameter in the interval $[0,1]$. Because species are distributed uniformly at random on the resource axis, a predator $j$ with range $r_{j}$ has on average $r_{j} S$ prey. The same applies to the reduced range $r_{j}^{\prime}$, and therefore a predator has $\Delta k=\left(r_{j}-r_{j}^{\prime}\right) S=(1-c) r_{j} S$ anticipated prey unaccounted for after the range reduction. To account for this, $\Delta k$ prey (rounded to the nearest integer value) are selected randomly from those species $i$ with niche value $n_{i} \leq n_{j}$ that are not already a prey of species $j$. The parameter $c$ is thus a measure of prey contiguity: for $c=0$, all prey of $j$ are selected randomly among species with $n_{i} \leq n_{j}$ and one recovers the generalized cascade model (Stouffer et al. 2005), whereas for $c=1$, all prey are contiguous and one recovers the niche model. Diet contiguity $c$ can be thought of as the degree to which a unidimensional niche axis is able to explain the properties of an empirical food web.

\section{Parameter estimation for model food webs}

For each empirical food web, we need to know three parameter values in order to generate food webs with the generalized niche model (Stouffer et al. 2006): the number of species $S$, the linkage density $z$, and the diet contiguity $c$. The number of species and the linkage density can be obtained directly from the empirical network. Therefore, only one parameter is estimated for each food web.

Estimating the diet contiguity $c$ is involved since in principle there is no direct way to measure it from a given food web; rather, $c$ needs to be estimated from the data (Stouffer et al. 2006). To do this, we use the following procedure for each food web:

1) Obtain 20 model food webs with each of three different values of $c \in\left\{0.5, c^{*}, 1.0\right\}$, where $c^{*}$ is the best estimate of $c$ obtained using the procedure proposed by Stouffer et al. (2006).
2) Estimate the $z$ score for different compartmentalization properties (modularity, trophic and sink homogeneity, number of compartments, standard deviation of the compartment sizes, and average size of compartments with more than one species), for the prey and predator views. Estimate the log-likelihood of each value of $c$ as the sum of squares of the $z$ scores.

3) Select the value of $c$ with the highest log-likelihood, generate new model food webs with values of $c$ around it, and iterate the process until the likelihood does not improve.

Note that, since this procedure to estimate $c$ is not exhaustive (exhaustive search is too expensive computationally given the large number of operations involved), we cannot rule out that there are slightly different values of $c$ that better explain the data. We list our best estimates for the parameters in Table 1.

\section{RESULTS \\ Compartment definition and identification}

As noted previously, compartmentalization refers to the existence of groups of species that have a higher probability of interacting with each another than with other species in the food web (May 1972, Girvan and Newman 2002, Krause et al. 2003). What constitutes an interaction, however, depends largely on the process under consideration. One possible interpretation would be that a group of species interacts strongly if there are many direct trophic interactions between them. A group of species can also interact strongly via competition, if they share a large number of prey, even though there might not be any direct trophic interactions between the species in the group.

With this in mind, we consider here three different definitions of a compartment in a food web (Methods): (1) a group of species that are densely connected to each other (density view); (2) a group of species that share a large number of prey (predator view); and (3) a group of species that share a large number of predators (prey view) (Fig. 1).

From a topological perspective, these are the only three sensible definitions of compartment since they 

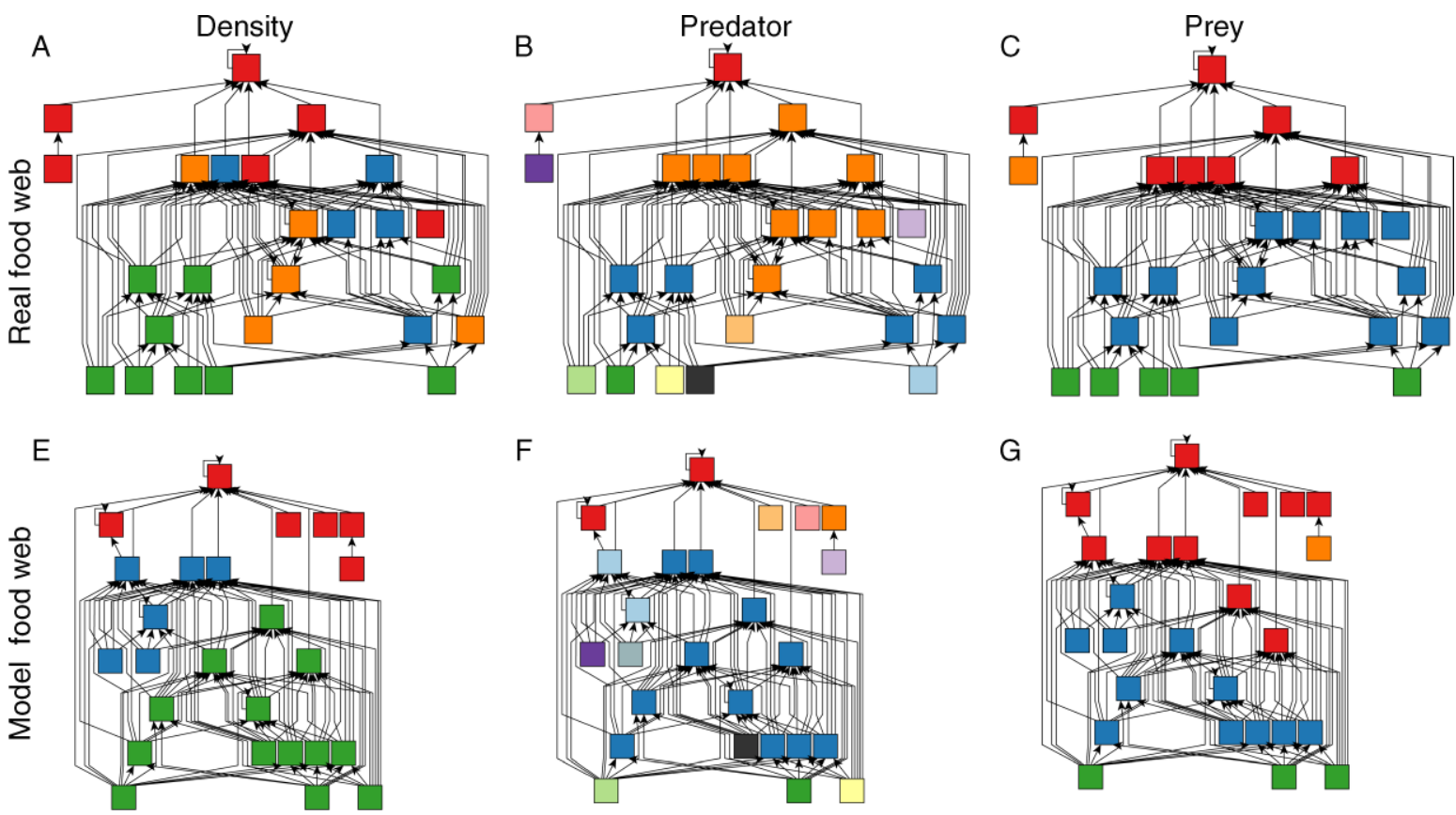

$\mathrm{F}$

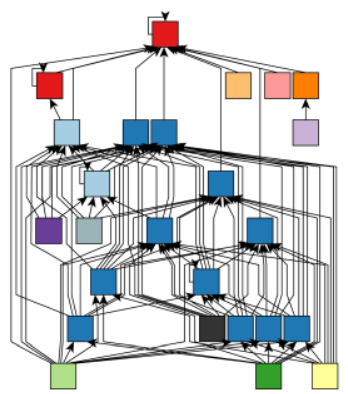

G

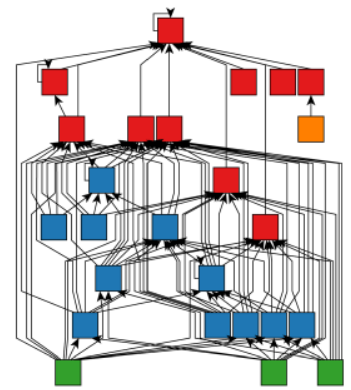

D) Real food web

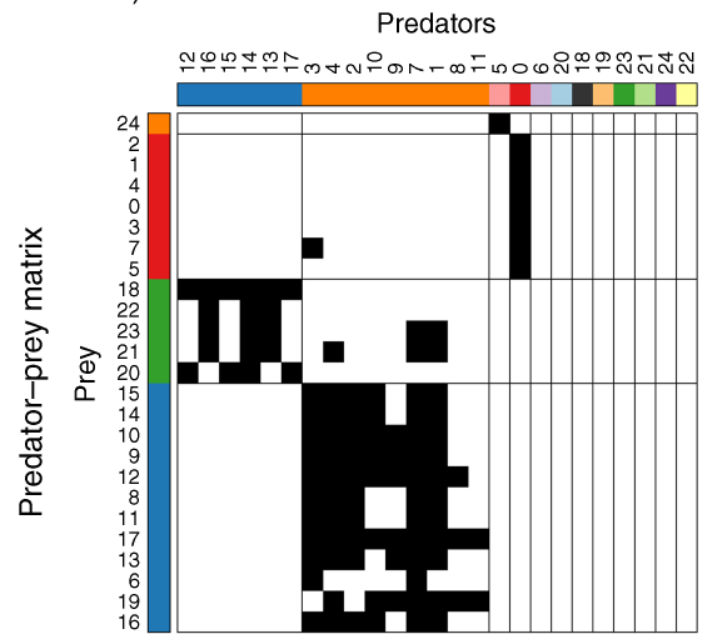

H) Model food web

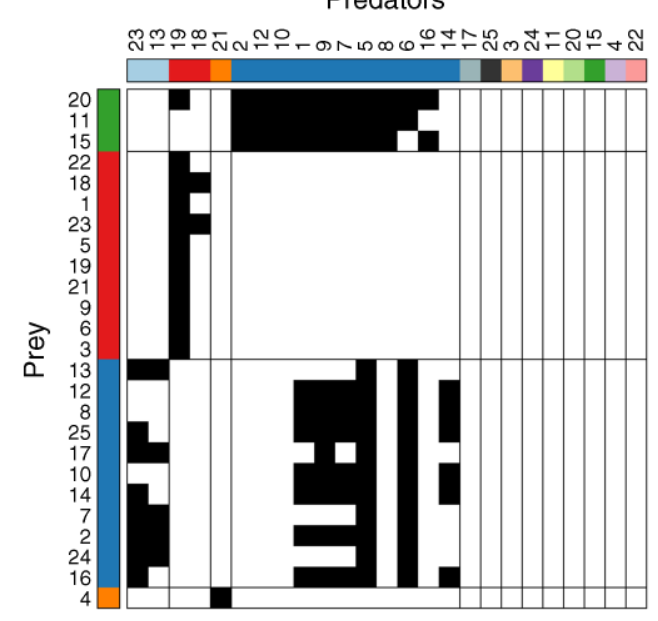

FIg. 1. Compartments in empirical and model food webs. (A-D) Bridge Brook Lake food web. (E-H) Typical generalized niche model of the Bridge Brook Lake food web. Nodes represent trophic species, and links indicate trophic interactions between species, with arrows pointing in the direction of mass flow (that is, from prey to predator). Different colors indicate different compartments according to the density view (A and E), the predator view (B and F), and the prey view (C and G). The three views yield distinct compartmentalizations. Compartments in the density view contain species that are densely interconnected to each other. Compartments in the prey (alternatively, predator) view correspond to groups of species with similar predators (prey). Combining these two views, the adjacency matrix of the food web (whose element in row $i$ and column $j$ represents the trophic interaction from prey $i$ to predator $j$ ) can be conveniently rearranged so that its block structure becomes apparent (D and H). Regardless of the view, compartments in the real and model webs have qualitatively similar properties (for example, compartment size and structure).

correspond to measures of compartmentalization based on all links, the incoming links, and the outgoing links of each species, respectively. The density view is the appropriate generalization to directed networks (Arenas et al. 2007, Leicht and Newman 2008) of algorithms that have been used before to identify compartments in food webs disregarding the direction of trophic interactions (Girvan and Newman 2002, Krause et al. 2003, Rezende et al. 2009). The prey and predator views result in compartments that are closely related to the groups defined by Allesina and Pascual (2009) (Fig. 1D, H). 
In the density view, the modularity function in Eq. 2 measures the difference between the fraction of interactions within compartments and the expected fraction of interactions within compartments in a random partition, taking into account the direction of the trophic interactions (Leicht and Newman 2008). In the predator (conversely, prey) view, we consider partitions based only on the prey (predators) of a species. In the predator (prey) view, the modularity in Eq. 3 is large when species in the same compartment share more prey (predators) than one would expect from chance alone (Guimerà et al. 2007).

We have analyzed the compartmentalized structure of 10 of the most complete food webs in the literature (Table 1 and Williams and Martinez [2008]) from each of these three viewpoints. Not surprisingly, the three views yield distinct compartmentalizations of a food web (Fig. 1A-D).

\section{Degree of compartmentalization of empirical food webs}

Recent studies have observed correlations between species characteristics and compartmentalization in food webs: species within compartments seem to have similar habitats (Girvan and Newman 2002, Krause et al. 2003) and seem to be related phylogenetically (Rezende et al. 2009). However, none of these studies has evaluated quantitatively the extent to which the various factors can actually explain the observed compartmentalization. In fact, quantitative investigations of compartmentalization have only been able to establish that real food webs are more compartmentalized than would be expected for a randomly assembled food web (Krause et al. 2003, Rezende et al. 2009).

Here, we take a more systematic approach and proceed by investigating empirical compartmentalization vis-à-vis that of two of the simplest models known to generate realistic food webs (rather than purely random networks): the niche model of Williams and Martinez (2000) and its generalization by Stouffer et al. (2006). By understanding the cause of compartmentalization in the models, we gain insights into the causes of compartmentalization in real food webs.

We present here the results for the generalized niche model while those for the niche model are shown in Appendix E. Specifically, we compare modularity values for the real food webs to those obtained from the generalized niche model, for each of the three views. As we show in Fig. 2A-C, the modularity of empirical food webs is within the $99 \%$ expectation interval of the models in 28 out of 30 cases, regardless of the compartment definition we use. In the two remaining cases, the modularity of the empirical food web is only slightly below the model predictions. Thus, real food webs are never more compartmentalized than one would expect from the generalized niche model.

Beyond modularity, we have also measured a variety of other properties of the compartments (Appendix D: Fig. D1): the number of compartments, the standard deviation of the number of species per compartment, and the mean size of compartments containing more than one species. For all of these properties and, again, for each definition of compartment, almost all the points (88 out of 90) fall within the $99 \%$ expectation intervals.

In general, the original niche model performs worse than the generalized niche model (see Appendix E). This is largely due to its tendency to overestimate the modularity of empirical networks.

\section{Trophic structure of empirical and model compartments}

In the previous section we have shown that the degree of compartmentalization of empirical food webs is never larger than expected from the generalized niche model. Moreover, we have shown that other properties of real compartments (the number of compartments, the typical compartment size, and the standard deviation of the size of compartments) are also compatible with the predictions of the generalized niche model.

Ecologically, however, a far more important consideration is whether the trophic structure of real compartments is also the same as that of model compartments. To investigate this question, we examine two measures of compartment homogeneity (see Methods for details): trophic-level homogeneity and sink homogeneity. Trophic homogeneity measures to what extent species in the same compartment occupy similar trophic levels. Sink homogeneity measures to what extent species in the same compartment belong to the same set of sink food webs.

In Fig. 2D-I, we show the homogeneity of compartments identified in our model networks using the three compartment identification approaches. For all views, mean trophic-level homogeneity is typically higher than expected from chance alone, suggesting that trophic level is a strong driver of compartmentalization. Mean sink homogeneity, by contrast, is significantly positive only in the density view, and random or even negative for the predator and prey views (since compartments in these two views cut across food chains).

Importantly, Fig. 2 also shows that, like for the number of compartments and their sizes, the generalized niche model accounts for the trophic structure of the compartments: in only two cases out of 60 is the observed homogeneity smaller than expected from the model. As before, the original niche model tends to less accurately explain the trophic structure of empirical compartments (see Appendix E).

\section{Empirical compartmentalization and niche space}

The previous sections suggest that the compartmentalization observed in real food webs can be accounted for by some mechanism that is already encoded in the generalized niche model. This result is counterintuitive because the generalized niche model does not incorporate attributes - such as habitat fragmentation or phylogenetics - that would explicitly give rise to com- 


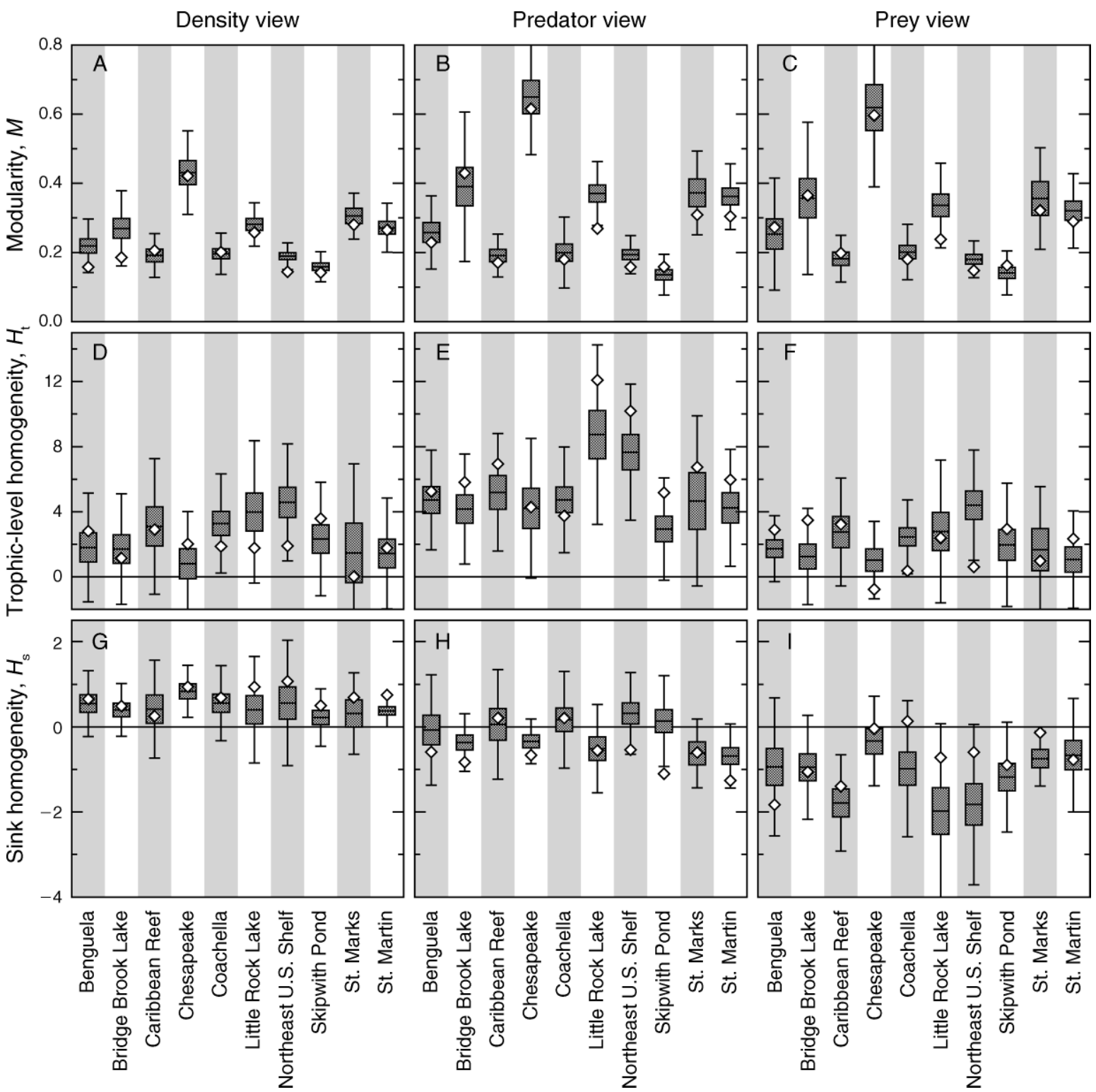

FIG. 2. Degree of compartmentalization and trophic structure of compartments for empirical and generalized niche model food webs. Compartments are defined according to (A, D, G) the density view, (B, E, H) the predator view, and (C, F, I) the prey view. (A-C) Modularity; (D-F) trophic homogeneity; (G-I) sink homogeneity. Symbols indicate empirical values. Shaded boxes with error bars indicate model values, with the shaded box indicating the $99 \%$ confidence interval for the mean and the error bars indicating the $99 \%$ confidence interval for the values (see Appendix B for details on how we estimate the confidence intervals). Despite the different food webs having very distinct values of modularity, empirical modularities are never larger than predicted by the generalized niche model $(\mathrm{A}-\mathrm{C})$. Trophic homogeneity is highest in compartments derived from the predator view (D-F), and sink homogeneity is highest in compartments derived from the density view (G-I). Empirically observed values are within the $99 \%$ confidence interval for 58 out of 60 cases, indicating that the trophic structure of empirical compartments is compatible with the predictions of the generalized niche model.

partments. Conceptually, the only relevant property of a species in the model is its niche value: other than the niche value of each species, the only parameters of the model are global parameters such as the number of species, the average number of prey (or predators) per species, and the diet contiguity. Therefore, compartments in generalized niche model food webs seem to arise from the underlying niche structure of the food web.

This conjecture has two important implications, which we investigate using the generalized niche model. First, if compartments arise from the niche structure underpinning the food web, compartmentalization should increase with increasing diet contiguity $c$ (see 


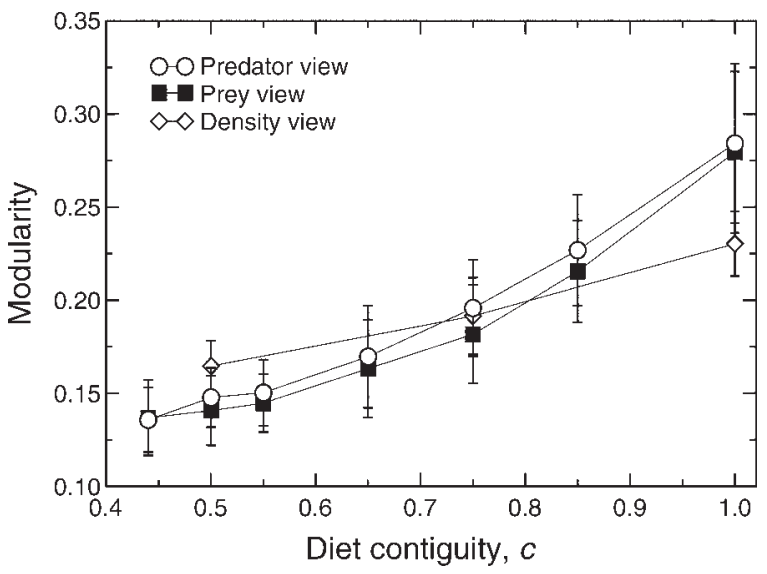

FIG. 3. Modularity as a function of diet contiguity for generalized niche models of the Caribbean Reef food web. Error bars indicate the standard deviation of the modularity. Increasing diet contiguity results in greater compartmentalization regardless of the definition of compartment. We obtain qualitatively similar results for all food webs studied (see Appendix F: Fig. F1).

Methods) because the niche values of species become more relevant when diets are continuous. The increased explanatory power of niche values is due to the fact that large contiguity of diets in the model induces strong correlations because two species with similar niche values are also expected to have similar predators (and, to a lesser extent, prey). Indeed, we observe that, regardless of the compartment definition, increasing diet contiguity in the generalized niche model results in greater compartmentalization (Fig. 3 and Appendix F). The original niche model's overestimation of compartmentalization appears to stem from this fact as diet contiguity in this model is maximal.

Second, if compartments arise from the niche structure, then one would expect the niche to be a strong driver for compartmentalization and compartments to have a strong "niche signal." To investigate this, we examine a niche homogeneity that quantifies to what extent species in the same compartment have similar niche values (see Methods for details). In Fig. 4, we show that compartments in all views are typically homogeneous in terms of the niches of the species they comprise, in agreement with our expectation.

\section{DiscusSION}

Our study is in agreement with previous studies in showing that empirical food webs are significantly more compartmentalized than purely structureless networks (Krause et al. 2003, Rezende et al. 2009). We extend these studies by considering alternative definitions of compartment (all sensible definitions from a network perspective, including "regular" compartmentalization as well as the groups introduced by Allesina and Pascual (2009)) and by relating the observed compartmentalization to species attributes across multiple food webs from a variety of environments.

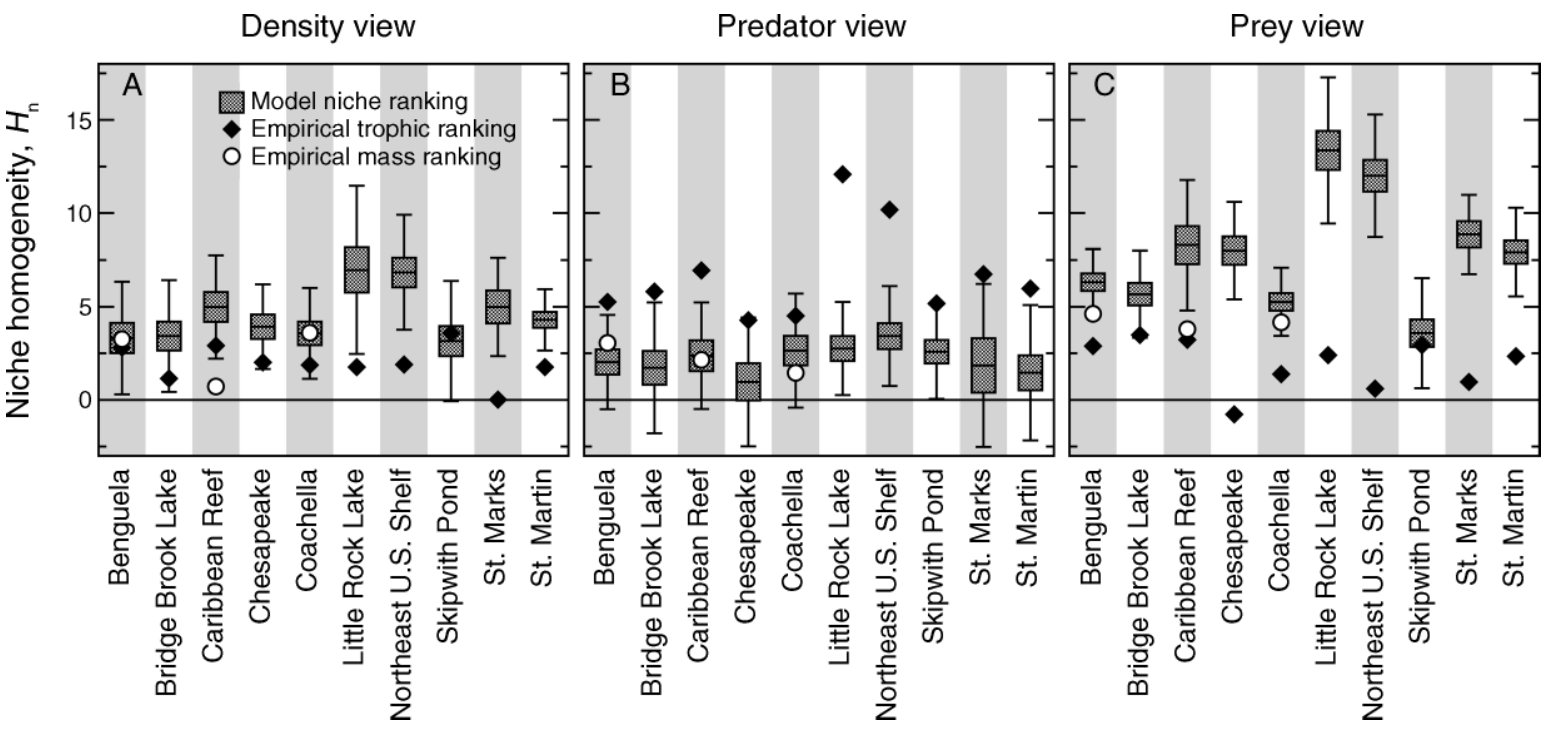

FIG. 4. Niche homogeneity of compartments in the generalized niche model: trophic level and mass as empirical proxies of niche value. (A) Density view; (B) predator view; (C) prey view. Shaded boxes with error bars indicate model values, with the shaded box indicating the $99 \%$ confidence interval for the mean and the error bars indicating the $99 \%$ confidence interval for the values. Confidence intervals are calculated using the $z$ score (see Appendix B for details). Niche homogeneity is highest in compartments derived from the prey view but has positive means in all views, which indicates that niche is a strong driver for compartmentalization. Black diamonds indicate the empirical trophic homogeneity, and white circles indicate the empirical mass homogeneity in mass-resolved food webs. Note that trophic homogeneity in the empirical webs consistently lies outside the range of niche homogeneity predicted by the model, suggesting that trophic level is a poor proxy for niche value. Though the data are limited for the food webs we consider, mass appears to be a far better proxy. 
Furthermore, by relating our empirical results to those for model food webs, we are able to (1) demonstrate that the generalized niche model generates food webs with compartmentalization compatible with what is observed empirically and (2) identify mechanisms that are sufficient to quantitatively explain all the compartmentalization observed in real food webs. Compartmentalization in the generalized niche model is an emergent phenomenon, arising as a consequence of species' diet contiguity.

This conclusion has practical, theoretical, and methodological implications. First, it clarifies that compartmentalization can be explained solely by niche-value ranking of species. This implies that compartmentalization does not need to be explicitly introduced in our efforts to model either ecosystem structure or ecosystem dynamics. In this regard, our results need to be analyzed vis-à-vis the work by Allesina and Pascual (2009), which argues that group-based models are more appropriate than niche-based models like the generalized niche model and calls for explicitly incorporating groups in our efforts to model food-web structure. While recognizing that we need more work to properly address the apparent contradiction between these results, we note that:

1) As we have shown using the prey and predator views, the relevant predator and prey groups (and therefore, presumably, those defined by Allesina and Pascual 2009) have uniform niche values, so niche has substantial explanatory power, in any case.

2) Our approach suggests that, if anything, the modularity of the generalized niche model (and definitely that of the original niche model) is slightly larger than that of some real food webs. In fact, group-based models might be a better fit to some real food webs precisely because, in those cases, the niche model is too compartmentalized; ultimately, the group-based model would provide the best fit to a totally random network, for example.

3) Model selection using likelihood approaches and information criteria is a powerful method but is not exempt of problems. In particular, the Akaike information criterion used by Allesina and Pascual (2009) is known to overestimate the optimal number of parameters of models and thus could be biased towards favoring group-based models over niche-based models.

From a theoretical perspective our results may help us see ecological perturbations in a new light. On the one hand, we find that compartmentalization increases with diet contiguity (Fig. 3) and increased compartmentalization may increase ecosystem stability by containing certain perturbations and preventing them from becoming system-wide (May 1972, Melián and Bascompte 2002). According to current knowledge, the invasion of a generalist predator, for example, would be assumed to reduce compartmentalization whereas the extinction of a generalist predator would be assumed to have the opposite effect. We demonstrate here, however, that how that predator's diet conforms to the community's niche space may be equally as important. Thus, perturbations that reduce diet contiguity may have cascading effects and may be more likely to be catastrophic. On the other hand, perturbations that increase diet contiguity may directly lead to food-web fragmentation. Interestingly, by rephrasing the issue in terms of diet contiguity, as opposed compartmentalization, we move from a feature that must be measured at the community scale to one that can be assessed on a species-specific basis.

It is important to recognize that without a true empirical analogue to niche value our results can only provide indirect evidence for the origin of food-web compartmentalization in real ecosystems. In this sense, our results are complementary to the empirical results reported for the Caribbean food web (Rezende et al. 2009). We note, also, that our approach can be used to quantitatively test the suitability of potential proxies for niche value; since compartments are homogeneous in terms of the niches of the species they contain (Fig. 4), the analysis of the composition of real compartments can help us answer quantitatively the long-standing question of what determines the ecological niche of a species.

Indeed, if we have reason to believe that any given empirical property $\mathrm{X}$ serves as a good proxy for niche value, then the homogeneity of $\mathrm{X}$ in empirical networks should be consistent with the niche homogeneity of model-generated food webs. In Fig. 4, we use this approach to investigate whether trophic level or species' mass are good proxies. We find that trophic-level homogeneity in the empirical webs lies consistently outside the range of niche homogeneity predicted by the model (particularly in the predator and prey views) and trophic level thus appears to be a poor proxy for niche value. Data on species' masses are limited for the food webs we consider, but those data that are available suggest that mass is a considerably better proxy. These results are in agreement with others in the literature (Jennings et al. 2002, Woodward et al. 2005), and our analysis provides a novel way to quantitatively test any potential proxy for niche value.

\section{ACKNOWLEDGMENTS}

We thank J. Bascompte, J. Duch, P. D. McMullen, R. D. Malmgren, A. L. Salazar, E. N. Sawardecker, S. M. D. Seaver, I. Sirer, and M. J. Stringer for comments and suggestions. D. B. Stouffer acknowledges the support of a CSIC JAE Postdoctoral Fellowship. M. E. J. Newman acknowledges support from NSF grant DMS-0804778 and the James S. McDonnell Foundation. L. A. N. Amaral acknowledges the support of NSF award SBE 0624318, and of the W. M. Keck Foundation.

\section{Literature Cited}

Allesina, S., D. Alonso, and M. Pascual. 2008. A general model for food web structure. Science 320:658-661.

Allesina, S., and M. Pascual. 2009. Food web models: a plea for groups. Ecology Letters 12:652-662. 
Arenas, A., A. Díaz-Guilera, and C. J. Pérez-Vicente. 2006. Synchronization reveals topological scales in complex networks. Physical Review Letterrs 96:114102.

Arenas, A., J. Duch, A. Fernández, and S. Gómez. 2007. Size reduction of complex networks preserving modularity. New Journal of Physics 9:article number 176.

Baird, D., and R. E. Ulanowicz. 1989. The seasonal dynamics of the Chesapeake Bay ecosystem. Ecological Monographs 59:329-364.

Bascompte, J., and P. Jordano. 2007. Plant-animal mutualistic networks: the architecture of biodiversity. Annual Review of Ecology, Evolution, and Systematics 38:567-593.

Bascompte, J., and C. J. Melián. 2005. Simple trophic modules for complex food webs. Ecology 86:2868-2873.

Camacho, J., R. Guimerà, and L. A. N. Amaral. 2002. Robust patterns in food web structure. Physical Review Letters 88: 228102.

Camacho, J., D. B. Stouffer, and L. A. N. Amaral. 2007. Quantitative analysis of the local structure of food webs. Journal of Theoretical Biology 246:260-268.

Cattin, M.-F., L.-F. Bersier, C. Banašek-Richter, R. Baltensperger, and J.-P. Gabriel. 2004. Phylogenetic constraints and adaptation explain food-web structure. Nature 427:835839.

Christian, R. R., and J. J. Luczkovich. 1999. Organizing and understanding a winter's seagrass foodweb network through effective trophic levels. Ecological Modelling 117:99-124.

Cohen, J. E. 1977. Food webs and the dimensionality of trophic niche space. Proceedings of the National Academy of Sciences USA 74:4533-4536.

Cohen, J. E., F. Briand, and C. M. Newman. 1990. Community food webs: data and theory. Springer-Verlag, Berlin, Germany.

Danon, L., A. Díaz-Guilera, J. Duch, and A. Arenas. 2005. Comparing community structure identification. Journal of Statistical Mechanics: Theory and Experiment 2005(9):P09008.

Dunne, J. A., R. J. Williams, and N. D. Martinez. 2002. Foodweb structure and network theory: the role of connectance and size. Proceedings of the National Academy of Sciences USA 99:12917-12922.

Fortunato, S., and M. Barthélemy. 2007. Resolution limit in community detection. Proceedings of the National Academy of Sciences USA 104:36-41.

Girvan, M., and M. E. J. Newman. 2002. Community structure in social and biological networks. Proceedings of the National Academy of Sciences USA 99:7821-7826.

Goldwasser, L., and J. Roughgarden. 1993. Construction of a large Caribbean food web. Ecology 74:1216-1233.

Guimerà, R., and L. Amaral. 2005. Functional cartography of complex metabolic networks. Nature 433:895-900.

Guimerà, R., M. Sales-Pardo, and L. Amaral. 2007. Module identification in bipartite and directed networks. Physical Review E 76:036102.

Havens, K. 1992. Scale and structure in natural food webs. Science 257:1107-1109.

Jennings, S., J. K. Pinnegar, N. V. C. Polunin, and K. J. Warr. 2002. Linking size-based and trophic analyses of benthic community structure. Marine Ecology Progress Series 226: 77-85.

Kondoh, M. 2008. Building trophic modules into a persistent food web. Proceedings of the National Academy of Sciences USA 105:16631-16635.

Krause, A. E., K. A. Frank, D. M. Mason, R. E. Ulanowicz, and W. W. Taylor. 2003. Compartments revealed in foodweb structure. Nature 426:282-285.

Leicht, E. A., and M. E. J. Newman. 2008. Community structure in directed networks. Physical Review Letters 100: 118703.
Levine, S. 1980. Several measures of trophic structure applicable to complex food webs. Journal of Theoretical Biology 83:195-207.

Link, J. 2002. Does food web theory work for marine ecosystems? Marine Ecology Progress Series 230:1-9.

Martinez, N. D. 1991. Artifacts or attributes? Effects of resolution on the Little Rock Lake food web. Ecological Monographs 61:367-392.

May, R. M. 1972. Will a large complex system be stable? Nature 238:413-414.

Melián, C. J., and J. Bascompte. 2002. Complex networks: two ways to be robust? Ecology Letters 5:705-708.

Newman, M. E. J., and M. Girvan. 2004. Finding and evaluating community structure in networks. Physical Review E 69:026113.

Ng, C. A., M. B. Berg, D. J. Jude, J. Janssen, P. M. Charlebois, L. A. N. Amaral, and K. A. Gray. 2008. Chemical amplification in an invaded food web: Seasonality and ontogeny in a high biomass, low diversity ecosystem. Environmental Toxicology and Chemistry 27:2186-2195.

Opitz, S. 1996. Trophic interactions in Caribbean coral reefs. Technical Report 43. International Center for Living Aquatic Resources Management (ICLARM), Manila, Philippines.

Pascual, M., and J. A. Dunne, editors. 2006. Ecological networks: linking structure to dynamics in food webs. Oxford University Press, Oxford, UK.

Pimm, S. L. 1979. The structure of food webs. Theoretical Population Biology 16:144-158.

Pimm, S. L. 2002. Food webs. First edition. University of Chicago Press, Chicago, Illinois, USA.

Pimm, S. L., and J. H. Lawton. 1980. Are food webs divided into compartments? Journal of Animal Ecology 49:879-898.

Polis, G. A. 1991. Complex trophic interactions in deserts: an empirical critique of food-web theory. American Naturalist 138:123-155.

Rezende, E. L., E. M. Albert, M. A. Fortuna, and J. Bascompte. 2009. Compartments in a marine food web associated with phylogeny, body mass, and habitat structure. Ecology Letters 12:779-788.

Sales-Pardo, M., R. Guimerà, A. A. Moreira, and L. Amaral. 2007. Extracting the hierarchical organization of complex systems. Proceedings of the National Academy of Sciences USA 104:15224-15229.

Srinivasan, U. T., J. A. Dunne, J. Harte, and N. D. Martinez. 2007. Response of complex food webs to realistic extinction sequences. Ecology 88:671-682.

Stouffer, D. B., J. Camacho, and L. A. N. Amaral. 2006. A robust measure of food web intervality. Proceedings of the National Academy of Sciences USA 103:19015-19020.

Stouffer, D. B., J. Camacho, R. Guimerà, C. A. Ng, and L. A. N. Amaral. 2005. Quantitative patterns in the structure of model and empirical food webs. Ecology 86:1301-1311.

Stouffer, D. B., J. Camacho, W. Jiang, and L. A. N. Amaral. 2007. Evidence for the existence of a robust pattern of prey selection in food webs. Proceedings of the Royal Society B 274:1931-1940.

Stouffer, D. B., C. A. Ng, and L. A. N. Amaral. 2008. Ecological engineering and sustainability: a new opportunity for chemical engineers. AIChE Journal 54:3040-3047.

Sugihara, G. 1984. Graph theory, homology, and food webs. Pages 83-101 in S. A. Levin, editor. Population biology. Volume 30 of Proceedings of Symposia in Applied Mathematics. American Mathematical Society, Providence, Rhode Island, USA.

Teng, J., and K. S. McCann. 2004. Dynamics of compartmented and reticulate food webs in relation to energetic flows. American Naturalist 164:85-100.

Warren, P. H. 1989. Spatial and temporal variation in a freshwater food web. Oikos 55:299-311. 
Williams, R. J., and N. D. Martinez. 2000. Simple rules yield complex food webs. Nature 404:180-183.

Williams, R. J., and N. D. Martinez. 2008. Success and its limits among structural models of complex food webs. Journal of Animal Ecology 77:512-519.

Woodward, G., B. Ebenman, M. Emmerson, J. M. Montoya, J. M. Olesen, A. Valido, and P. H. Warren. 2005. Body size in ecological networks. Trends in Ecology and Evolution 20: 402-409.

Yodzis, P. 1982. The compartmentation of real and assembled ecosystems. American Naturalist 120:551-570.

Yodzis, P. 1998. Local trophodynamics and the interaction of marine mammals and fisheries in the Benguela ecosystem. Journal of Animal Ecology 67:635-658.

\section{APPENDIX A}

Homogeneity calculations (Ecological Archives E091-207-A1).

\section{APPENDIX B}

Confidence intervals for compartment properties (Ecological Archives E091-207-A2).

\section{APPENDIX C}

The generalized niche model (Ecological Archives E091-207-A3).

\section{APPENDIX D}

Compartment properties for the generalized niche model (Ecological Archives E091-207-A4).

\section{APPENDIX E}

Compartment properties for the niche model (Ecological Archives E091-207-A5).

\section{APPENDIX F}

Modularity as a function of diet contiguity for all food webs (Ecological Archives E091-207-A6). 